\title{
Efeito da prática do futebol nas funções executivas de crianças e adolescentes. Um estudo de revisão sistemática
}

\author{
Effect of soccer practice on the executive functions of children and adolescents. A systematic review \\ study \\ Efecto de la práctica del fútbol en las funciones ejecutivas de niños y adolescentes. Un estudio de \\ revisión sistemática
}

\section{Resumo}

Introdução: A prática esportiva se inicia entre a infância e adolescência com o objetivo voltado a aprendizagem motora. A prática do futebol gera efeitos benéficos nas habilidades físicas e motoras. Estudos recentes vêm demonstrando o potencial da prática do futebol na infância para estimular o desenvolvimento cognitivo. Objetivo: Verificar por meio da revisão sistemática os efeitos da prática do futebol para as crianças e adolescentes nos domínios cognitivos e motores. Métodos: Foram feitas buscas nas bases de dados SciELO e PubMed, utilizando os termos (soccer OR futebol) associados a (executive functions OR funções executivas), (cognitive functions OR funções cognitivas) associadas a (soccer OR futebol) de 2010 a 2020, foram encontrados 402 artigos. Após critérios de exclusão, restaram nove artigos. Resultados: Os componentes mais avaliados, foram as funções executivas, que aparecem nos 9 estudos e a atenção seletiva que foi investigado em 6 trabalhos. Os artigos selecionados obtiveram uma amostra de 1069 participantes. Oito trabalhos associaram as funções executivas à prática do futebol. Em seis trabalhos, observou-se que os atletas de 7 a 17 anos que treinavam futebol de forma profissional obtiveram melhores resultados nos testes cognitivos em comparação aos que treinavam em clubes amadores ou não treinavam o esporte. Conclusão: Os resultados indicam relação positiva na melhora da capacidade das funções executivas entre crianças e adolescentes que iniciaram a modalidade do futebol no período de latência.

Palavras-chave: Futebol; Funções executivas; Cognição; Crianças; Adolescentes. 


\begin{abstract}
Introduction: Sports practice begins between childhood and adolescence with the goal of motor learning. Football practice has beneficial effects on physical and motor skills. Recent studies have demonstrated the potential of childhood soccer practice to stimulate cognitive development. Objective: To verify, through a systematic review, the effects of soccer practice for children and adolescents in the cognitive and motor domains. Methods: SciELO and PubMed databases were searched using the terms (soccer OR soccer) associated with (executive functions OR executive functions), (cognitive functions OR cognitive functions) associated with (soccer OR soccer) from 2010 to 2020, 402 articles were found. After exclusion criteria, nine articles remained. Results: The most evaluated components were the executive functions, which appear in the 9 studies and the selective attention that was investigated in 6 studies. The selected articles obtained a sample of 1069 participants. Eight works associated executive functions to the practice of football. In six studies, it was observed that athletes from 7 to 17 years old who trained football professionally obtained better results in cognitive tests compared to those who trained in amateur clubs or did not train the sport. Conclusion: The results indicate a positive relationship in the improvement of the capacity of executive functions among children and adolescents who started soccer in the latency period.
\end{abstract}

Keywords: Soccer; Executive functions; Cognition; Children; Adolescents.

\title{
Resumen
}

Introducción: La práctica deportiva se inicia entre la infancia y la adolescencia con el objetivo del aprendizaje motor. La práctica del fútbol tiene efectos beneficiosos sobre las habilidades físicas y motoras. Estudios recientes han demostrado el potencial de la práctica del fútbol infantil para estimular el desarrollo cognitivo. Objetivo: Verificar, a través de una revisión sistemática, los efectos de la práctica del fútbol en niños y adolescentes en los dominios cognitivo y motor. Métodos: Se realizaron búsquedas en las bases de datos SciELO y PubMed utilizando los términos (soccer OR soccer) asociados con (funciones ejecutivas OR funciones ejecutivas), (funciones cognitivas OR funciones cognitivas) asociados con (soccer OR soccer) de 2010 a 2020, se encontraron 402 artículos. Después de los criterios de exclusión, quedaron nueve artículos. Resultados: Los componentes más evaluados fueron las funciones ejecutivas, que aparecen en los 9 estudios y la atención selectiva que se investigó en 6 estudios. Los artículos seleccionados obtuvieron una muestra de 1069 participantes. Ocho trabajos asocian funciones ejecutivas a la práctica del fútbol. En seis estudios se observó que los deportistas de 7 a 17 años que entrenaron fútbol profesionalmente obtuvieron mejores resultados en las pruebas cognitivas en comparación con los que entrenaron en clubes amateurs o no entrenaron el deporte. Conclusión: Los resultados indican una relación positiva en la mejora de la capacidad de las funciones ejecutivas entre los niños y adolescentes que iniciaron el fútbol en el período de latencia.

Palabras clave: Fútbol; Funciones ejecutivas; Cognición; Niños; Adolescentes.

\section{Introdução}

O período da infância e adolescência são os momentos com maior plasticidade e adaptação motora, física e cognitiva do desenvolvimento humano, durante esta fase do desenvolvimento acontece inúmeras consolidações neuro cognitivas e motoras (Diamond \& Lee, 2011; Diamond \& Ling, 2016; Swinny et al., 2005). Em vista dos processos adaptativos destes períodos do desenvolvimento, nota-se a necessidade de estímulos ambientais pertinentes a favorecer e estimular melhores condições de ganhos físicos, motores e cognitivos (Hoeboer J.2016). Dentre as inúmeras estratégias ambientais pertinentes a beneficiarem condições favoráveis ao desenvolvimento integral de crianças e adolescentes, encontra-se a prática esportiva (Becker et al., 2018; Diamond \& Lee, 2011; Ishihara et al., 2017a; Sigmundsson et al., 2017; van der Fels et al., 2015).

A prática esportiva vem demonstrando ao longo dos anos seus efeitos benéficos inerentes as capacidades motoras e físicas (Cardeal et al., 2013), também é digno de nota que estudos recentes veem apresentando suas ações em contribuir intimamente com o desenvolvimento das capacidades cognitivas, tanto em alterações estruturais, quanto funcionais (Ishihara et al., 2017b, 2017a; Jacobson \& Matthaeus, 2014; Rafaello Pinheiro Mazzoccante et al., 2019). Ao salientar o efeito funcional do esporte nos domínios cognitivos, estes estão relacionados aos componentes das funções executivas que são responsáveis por planejar, criar, organizar e monitorar nossas ações, as quais dividem-se em três componentes interligados, controle inibitório, memória de trabalho e flexibilidade cognitiva (Becker et al., 2018; Diamond \& Lee, 2011; Ishihara \& Mizuno, 2018).

Dentre os esportes amplamente praticados na população brasileira por crianças e adolescentes encontra-se o futebol, sua prática acontece nos centros esportivos e nas horas de lazer deste público (Miguel \& Temer, 2015). O futebol demonstra imensa capacidade de alterar de maneira benéfica a formação neuro estrutural e funcional deste público, devido as suas 
exigências táticas e técnicas com grande demanda neuropsicológica, perceptivo-cognitivas, motora e seus benefícios advindos da prática do exercício físico, como aumento da aptidão cardiorrespiratória e da coordenação motora. (Hillman et al., 2014; Rafaello P. Mazzoccante et al., 2020; Tsai et al., 2012)

Estudos com o objetivo de analisar o efeito do futebol na infância e adolescência nos domínios cognitivos é uma abordagem bem recente, os poucos estudos publicados demonstram a grande relevância da prática do futebol para o desenvolvimento físico, motor e cognitivo do público infantil e adolescente. Em sua predominância os estudos acerca do futebol abrangem as capacidades que os atletas necessitam possuir para obter destaque, e hoje, com isso o estudo vem com intuito de verificar e reunir dados em revisão sistemática sobre os efeitos da prática do futebol em crianças e adolescente nas funções executivas.

Portanto, está revisão tem como objetivo verificar através de revisão sistemática os efeitos da prática do futebol para as crianças e adolescentes nos domínios cognitivos e motores.

\section{Metodologia}

Trata-se de um estudo de revisão sistemática, com o objetivo de revisar em estudos descritivos e experimentais, com aplicabilidade do futebol em crianças e adolescentes nas funções executivas. Foram selecionados separadamente títulos e resumos para identificar artigos com base em critérios de inclusão. A seleção dos artigos se iniciou a partir da leitura dos títulos e resumos. Se necessário, os autores completaram a leitura do texto completo para verificar se atendia os critérios de inclusão. Artigos originais publicados na língua inglesa e portuguesa foram selecionados.

As bases de dados utilizadas para a busca de artigos foram PubMed e SciELO. Palavras-chave usadas nas pesquisas foram (soccer OR futebol) AND (executive functions OR funções executivas), (cognitive functions OR funções cognitivas) AND (soccer OR futebol). Foram selecionados apenas artigos de 2010 a 2020. Os critérios de inclusão foram: o futebol como parte do programa de intervenção física; a amostra composta por crianças e/ou adolescentes; a avaliação das Funções Executivas. Os critérios de exclusão foram: pesquisa que abordasse o uso de suplementos, apresentar problema físico que impedisse a participação nos testes físicos, estar fazendo uso de medicamentos que alterassem os sentidos ou a cognição.

\section{Resultados}

$\mathrm{Na}$ base de dados SciELO, foram encontrados apenas 2 artigos, porém, nenhum elegível. Na base PubMed, a busca dos termos futebol e soccer associados a cognitive function ou executive function associados aos operadores boleanos gerou 402 artigos. Após exclusão dos artigos duplicados e a aplicação dos critérios de inclusão, nove artigos foram elegíveis para análise.
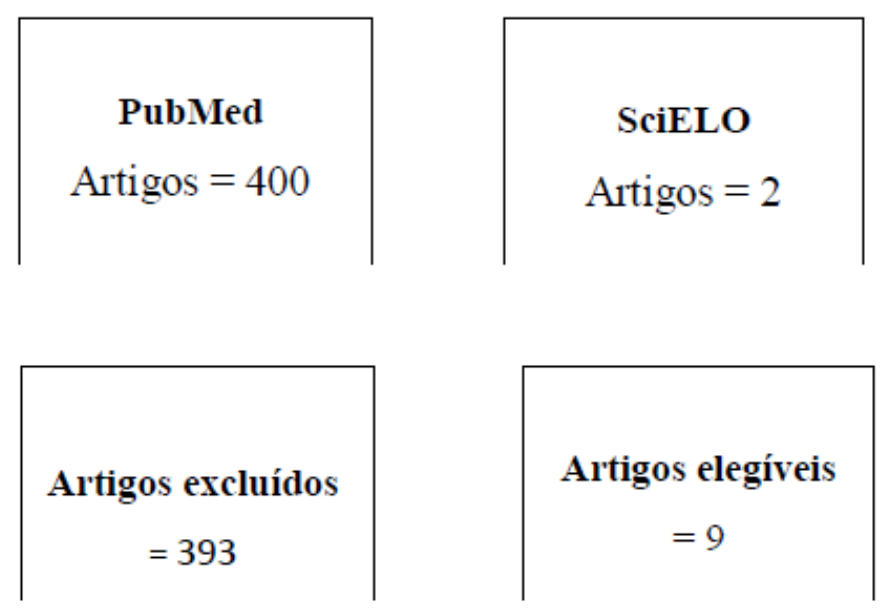


\section{Quadro 1.}

\begin{tabular}{|c|c|c|c|c|}
\hline Nome & Amostra & Medida e Instrumento & Intervenção & Principais Resultados \\
\hline Memmert et al. 2011 & $\begin{array}{c}112 \text { crianças entre } 7 \text { e } 13 \\
\text { anos }\end{array}$ & $\begin{array}{l}\text { Medidas - Habilidades de atenção, } \\
\text { pensamento criativo, funções } \\
\text { executivas. Instrumentos-Non- } \\
\text { specifc inattentional blindness task, } \\
\text { specific inattentional blindness } \\
\text { task, non-specific. }\end{array}$ & $\begin{array}{l}\text { Não houve intervenção. } \\
\text { Análise comparativa do } \\
\text { contexto não especifico e do } \\
\text { relacionamento ao esporte } \\
\text { em crianças. }\end{array}$ & $\begin{array}{l}\text { As crianças demostraram, à } \\
\text { medida que envelhecem, que } \\
\text { são capazes de produzir } \\
\text { números mais altos e soluções } \\
\text { mais originais e se tornam mais } \\
\text { flexiveis ao alternar entre } \\
\text { diferentes niveis de abstração. }\end{array}$ \\
\hline Verburgh et al. 2014 & $\begin{array}{l}126 \text { meninos com idades } \\
\text { entre } 8 \text { e } 12 \text { anos. } \\
84 \text { - Jogadores de elite; } \\
42 \text { - Jogadores } \\
\text { amadores; }\end{array}$ & $\begin{array}{l}\text { Medida - Funções executivas, QI, } \\
\text { atenção, estimativa de atividade } \\
\text { fisica. } \\
\text { Instrumento - Test stop signal, } \\
\text { tarefa adaptada desenvolvida por } \\
\text { Bergman-Nutley et al. Versão } \\
\text { modificada }\end{array}$ & $\begin{array}{l}\text { Não ouve intervenção. } \\
\text { Análise comparativa entre } \\
\text { grupo de jogadores de elite } \\
\text { e amadores }\end{array}$ & $\begin{array}{l}\text { Os jogadores de futebol de elite } \\
\text { superaram os amadores no } \\
\text { controle inibitório continuo e } \\
\text { em manter o estado de alerta. }\end{array}$ \\
\hline
\end{tabular}

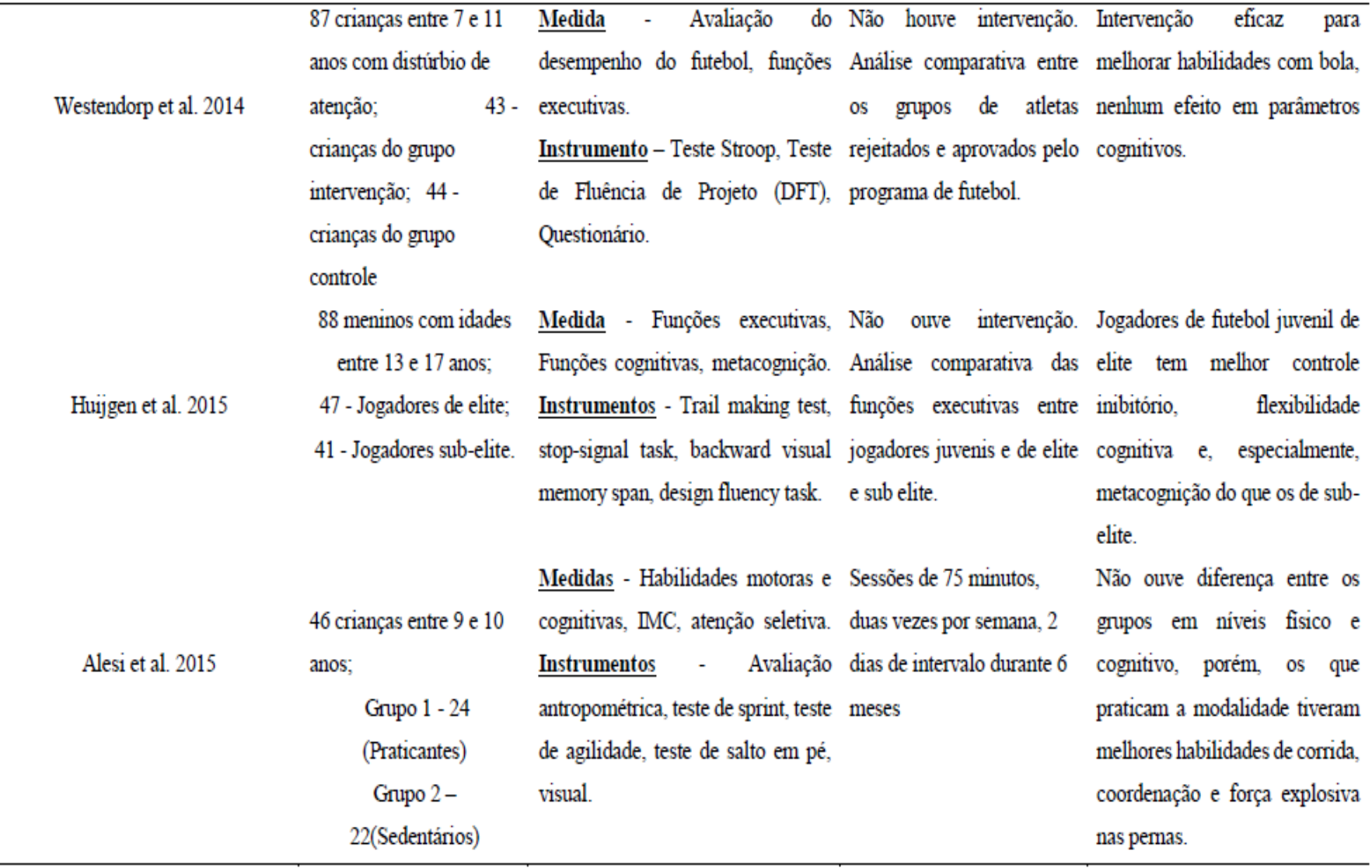




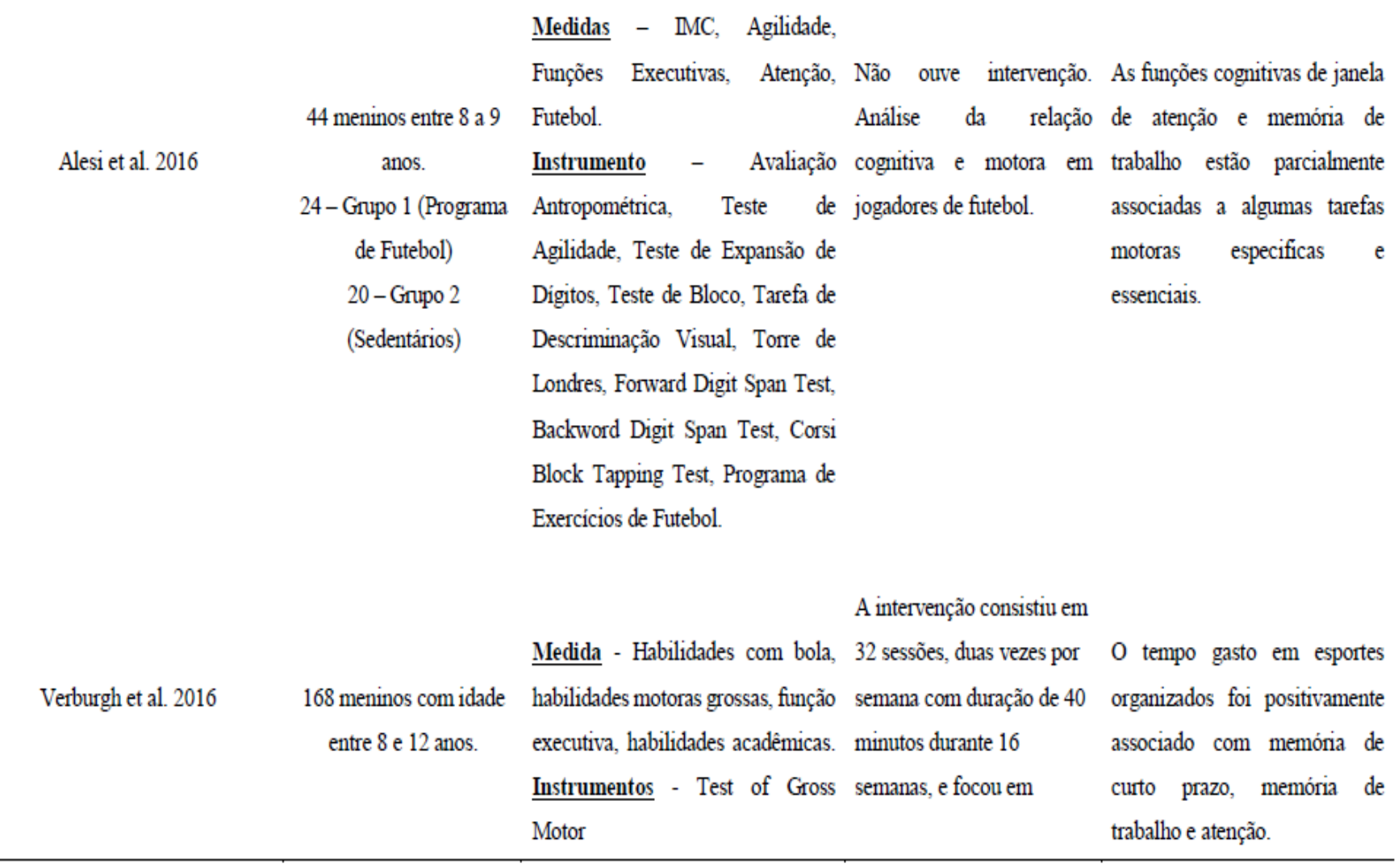

Fonte: Autores.

Os artigos selecionados obtiveram: amostra de á 1069 participantes, com faixas etárias entre 7 e 17 anos, 6 estudos transversais e 2 estudos longitudinais, com duração de 16 semanas a 6 meses, com frequência de 2 vezes semanais, com durações de 40 a 75 minutos. O componente mais avaliado foram as funções executivas, que aparecem em 9 trabalhos, atenção seletiva foi investigado em 6 trabalhos.

\section{Discussão}

Os estudos revisados apresentaram efeitos benéficos da prática do futebol para crianças e adolescentes, tanto nos estudos transversais, quanto nos longitudinais, o componente mais evidenciado nos estudos foram as funções executivas e atenção seletiva (Huijgen et al., 2015; Rafaello Pinheiro Mazzoccante et al., 2019; Souza et al., 2020; Verburgh et al., 2014).

Sakamoto. (2018) avaliaram as funções executivas entre os atletas do grupo de aprovados e o grupo de não aprovados por um programa de futebol com faixa etária entre 8 e 11 anos. O grupo de aprovados superou significativamente o grupo de rejeitados no test stroop e no desing fluency test. No questionário de avaliação de saúde mental o grupo de rejeitados apresentou melhores pontuações em resiliência do que o grupo de aprovados.

Verburgh. (2016) buscaram avaliar a relação entre atividade física, comportamento sedentário e funcionamento neurocognitivo com idades entre 8 e 12 anos. O tempo gasto em esportes foi responsável significativamente pela variação na inibição, memória de curto prazo, memória de trabalho e lapsos de atenção, onde mais tempo gasto em esportes foi associado a melhor desempenho. Em outro estudo Verburgh. (2014) realizaram uma análise comparativa entre grupo de jogadores de elite e amadores com idade de 8 e 12 anos. Os jogadores de futebol juvenil altamente talentosos superam os jogadores amadores na supressão de respostas motoras contínuas e na capacidade de atingir e manter um estado de alerta; ambos podem ser essenciais para o sucesso no futebol.8-9 
Huijgen. (Huijgen et al., 2015) avaliaram as funções executivas entre jogadores com idades entre 13 e 17 anos de elite e de sub-elite. Testes revelaram diferença significativa no nível acadêmico entre os grupos de elite e sub-elite. Os jogadores de futebol de elite superaram os amadores no controle inibitório, flexibilidade cognitiva e metacognição do que os de sub-elite. Achados similares de Westendorp. (2014) realizaram um programa de intervenção de futebol de 16 semanas. Nenhum efeito de intervenção foi encontrado nos parâmetros cognitivos. O grupo intervenção melhorou significativamente as habilidades com bola e com isso obtiveram melhora na resolução de problemas.

Memmert. (2011) realizou uma análise comparativa do contexto não específico e do relacionamento ao esporte em crianças com faixa etária de 7 e 13 anos. Foi demonstrado, que a medida que as crianças envelhecem, são capazes de produzir números mais altos e soluções mais originais e se tornam mais flexíveis ao alternar entre diferentes níveis de abstração.

O trabalho de Alesi. (2015) analisou habilidades motoras e cognitivas entre crianças de 9 a 10 anos que praticam futebol e crianças sedentárias utilizando um programa de treinamento de futebol. O programa de exercícios de futebol resultou em melhor corrida, coordenação e desempenho explosivo de força nas pernas e tempo de discriminação visual mais curto em crianças que frequentavam regularmente cursos de futebol, em comparação com os sedentários. Não ouve diferença entre os grupos em níveis físico e cognitivo.13

O estudo de Scharfen. (2019) analisou a relação cognitiva e motora em jogadores de futebol com faixa etária de 12 a 14 anos. Os testes indicaram que algumas funções cognitivas como janela de atenção e memória de trabalho, estão parcialmente associadas a algumas tarefas motoras.

Alesi. (2016) avaliaram os efeitos de um programa de treinamento de futebol com duração de 6 meses no grupo controle de crianças de 8 a 9 anos de idade, comparando a um grupo de crianças sedentárias. Após o programa de exercícios de futebol, houve diferenças significativas entre os grupos esportivos e sedentários nas habilidades de coordenação e funções executivas. O grupo controle apresentou ganhos significativamente maiores que o grupo de sedentários nas medidas de agilidade, memória operacional visuoespacial, atenção, planejamento e controle inibitório.

Os principais resultados do presente estudo obtidos através dos testes e das intervenções se dão pela demanda de trabalho exigida para uma partida de futebol, o ambiente, as ações exercidas, a interação com o grupo, a velocidade de raciocínio e processamento, são alguns dos fatores que exigem alta demanda cognitiva e motora. Logo, os adversos momentos do jogo podem levar a inúmeras reações, tomadas de decisão, alterar o estado de alerta, dentre outras inúmeras reações possíveis advindas de uma partida de futebol (Vertberg.2012).

As inúmeras reações experimentadas durante o jogo, vivenciadas desde a infância à adolescência, ocasionarão no desenvolvimento das funções executivas e cognitivas e motoras, dentre elas a atenção seletiva, memória de trabalho, controle inibitório, flexibilidade cognitiva, metacognição, melhor estado de alerta, e através delas serão capazes de produzir soluções mais originais e desenvolvimento do pensamento criativo. As funções executivas são importantes no futebol e podem até prever um futuro sucesso em jogadores de futebol (Vestberg.2012).

Poderíamos considerar a infância como o primeiro ato de prevenção primária do envelhecimento patológico e da perda de habilidades cognitivas, tendo em vista que o desenvolvimento cognitivo precoce será uma prevenção primária para retardar a poda neural na fase adulta (Schoentgen.2020). As funções executivas se bem estimuladas e trabalhadas em crianças e adolescentes serão de grande valia para a prática esportiva, tendo em vista a melhora no repertório motor e domínio cognitivo enriquecido, gerando melhor desempenho nas modalidades e podendo contribuir para demais atividades como atividades acadêmicas (Drollette et al., 2014).

O presente estudo demonstra que esse desafio seria um caminho para verificar e reunir dados em revisão sistemática sobre os efeitos da prática do futebol em crianças e adolescentes nas funções executivas nos poucos estudos existentes, para fins de pesquisas futuras. 


\section{Conclusão}

Por fim, os resultados indicam relação positiva na capacidade das funções executivas entre crianças e adolescentes que iniciaram a modalidade do futebol no período de latência e apresentam uma nova perspectiva aos profissionais de Educação Física e aos pais das crianças para atuação por meio de iniciação esportiva do público infantil, adequando o planejamento de aula não somente para os aspectos físicos, mas também cognitivos.

\section{Referências}

Becker, D. R., McClelland, M. M., Geldhof, G. J., Gunter, K. B., \& MacDonald, M. (2018). Open-Skilled Sport, Sport Intensity, Executive Function, and Academic Achievement in Grade School Children. Early Education and Development, 29(7), 939-955. https://doi.org/10.1080/10409289.2018.1479079

Cardeal, C. M., Pereira, L. A., Silva, P. F., \& França, N. M. (2013). Efeito de um programa escolar de estimulação motora sobre desempenho da função executiva e atenção em crianças Effect of school-based motor stimulation intervention on executive function and attention performance in children. $9(3)$, 4456. https://doi.org/10.6063/motricidade. 9(3).762

Diamond, A., \& Lee, K. (2011). Interventions shown to aid executive function development in children 4 to 12 years old. Science, 333(6045), 959-964. https://doi.org/10.1126/science. 1204529

Diamond, A., \& Ling, D. S. (2016). Conclusions about interventions, programs, and approaches for improving executive functions that appear justified and those that, despite much hype, do not. Developmental Cognitive Neuroscience, 18, 34-48. https://doi.org/10.1016/j.dcn.2015.11.005

Drollette, E. S., Scudder, M. R., Raine, L. B., Moore, R. D., Saliba, B. J., Pontifex, M. B., \& Hillman, C. H. (2014). Developmental Cognitive Neuroscience Acute exercise facilitates brain function and cognition in children who need it most: An ERP study of individual differences in inhibitory control capacity. Accident Analysis and Prevention, 7, 53-64. https://doi.org/10.1016/j.den.2013.11.001

Hillman, C. H., Pontifex, M. B., Castelli, D. M., Khan, N. A., Raine, L. B., Scudder, M. R., Drollette, E. S., Moore, R. D., Wu, C.-T., \& Kamijo, K. (2014). Effects of the FITKids Randomized Controlled Trial on Executive Control and Brain Function. Pediatrics, 134(4), e1063-e1071. https://doi.org/10.1542/peds.2013-3219

Huijgen, B. C. H., Leemhuis, S., Kok, N. M., Verburgh, L., Oosterlaan, J., Elferink-Gemser, M. T., \& Visscher, C. (2015). Cognitive functions in elite and sub-elite youth soccer players aged 13 to 17 years. PLoS ONE, 10(12), 1-13. https://doi.org/10.1371/journal.pone.0144580

Ishihara, T., \& Mizuno, M. (2018). Effects of tennis play on executive function in 6-11-year-old children: a 12-month longitudinal study. European Journal of Sport Science, 18(5), 741-752. https://doi.org/10.1080/17461391.2018.1444792

Ishihara, T., Sugasawa, S., Matsuda, Y., \& Mizuno, M. (2017a). Improved executive functions in 6-12-year-old children following cognitively engaging tennis lessons. Journal of Sports Sciences, 35(20), 2014-2020. https://doi.org/10.1080/02640414.2016.1250939

Ishihara, T., Sugasawa, S., Matsuda, Y., \& Mizuno, M. (2017b). Relationship between sports experience and executive old children : independence from physical fitness and moderation by gender. Developmental Science, January, 1-13. https://doi.org/10.1111/desc.12555

Jacobson, J., \& Matthaeus, L. (2014). Athletics and executive functioning: How athletic participation and sport type correlate with cognitive performance. Psychology of Sport and Exercise, 15(5), 521-527. https://doi.org/10.1016/j.psychsport.2014.05.005

Mazzoccante, Rafaello P., Corrêa, H. L., Sousa, B. R. C. de, Souza, I. R. C. de, Ribeiro, H. S., Ferreira, A. P., \& Melo, G. F. de. (2020). Artigo de Original A capacidade preditiva de indicadores de composição corporal, aptidão aeróbia e coordenação motora sobre a atenção e função executiva em crianças de 6 a 11 anos The predictive capacity of anthropometric indicators , body composition ,. R. Bras. Ci. e Mov, 26(2), 60-68.

Mazzoccante, Rafaello Pinheiro, Corrêa, H. D. L., Queiroz, J. L. De, Sousa, R. C. De, Raquel, I., Sousa, C. De, \& Barboza, M. A. (2019). ARTIGO ORIGINAL A relação da prática esportiva com o desempenho motor, atenção seletiva, flexibilidade cognitiva e velocidade de processamento em crianças de 7 a 10 anos. 29(3), 365-372.

Miguel, M., \& Temer, E. (2015). P ráticas dE E sporte e A tividade F ísica.

Sigmundsson, H., Englund, K., \& Haga, M. (2017). Associations of Physical Fitness and Motor Competence with Reading Skills in 9- and 12-Year-Old Children: A Longitudinal Study. SAGE Open, 7(2). https://doi.org/10.1177/2158244017712769

Souza, W. S. de S., Vinícius da Silva Pita, Corrêa, H. de L., SOUSA, I. R. C. de, \& Mazzoccante, R. P. (2020). Função executiva, coordenação motora e composição corporal, na infância: o papel da prática esportiva Executive. Research, Society and Development, 2020(9), 1-14.

Swinny, J. D., van der Want, J. J. L., \& Gramsbergen, A. (2005). Cerebellar development and plasticity: Perspectives for motor coordination strategies, for motor skills, and for therapy. Neural Plasticity, 12(2-3), 153-160. https://doi.org/10.1155/NP.2005.153

Tsai, C., Wang, C., \& Tseng, Y. (2012). Brain and Cognition Effects of exercise intervention on event-related potential and task performance indices of attention networks in children with developmental coordination disorder. Brain and Cognition, 79(1), 12-22. https://doi.org/10.1016/j.bandc.2012.02.004

van der Fels, I. M. J., te Wierike, S. C. M., Hartman, E., Elferink-Gemser, M. T., Smith, J., \& Visscher, C. (2015). The relationship between motor skills and cognitive skills in 4-16 year old typically developing children: A systematic review. Journal of Science and Medicine in Sport, 18(6), 697-703. https://doi.org/10.1016/j.jsams.2014.09.007

Verburgh, L., Scherder, E. J. A., Van Lange, P. A. M., \& Oosterlaan, J. (2014). Executive functioning in highly talented soccer players. PLoS ONE, 9(3). https://doi.org/10.1371/journal.pone.0091254. 